\title{
Dynamic Response of a Timoshenko Beam Subjected to a Moving Load on a Foundation with Hysteretic Damping
}

\author{
Desmond Adair ${ }^{1}$, Aigul Nagimova ${ }^{1}$, Martin Jaeger ${ }^{2}$ \\ ${ }^{1}$ Nazarbayev University \\ School of Engineering \& Digital Sciences, Nur-Sultan, 010000, Republic of Kazakhstan \\ dadair@nu.edu.kz; aigul.nagimova@nu.edu.kz \\ ${ }^{2}$ University of Tasmania \\ College of Sciences \& Engineering, Hobart, TAS 7001, Australia \\ martin.jaeger@utas.edu.au.
}

\begin{abstract}
For many studies concerning foundation, such as rails and pavements, the mechanism for energy dissipation within a foundation is considered only to be by viscous behaviour. It is generally considered however that dissipation of energy in soil-type foundations takes place, in the main, due to internal friction damping, known as hysteretic damping. Also quite often the simpler EulerBernoulli beam formulation is used, hence excluding the effects of shear rigidity and radius of gyration of the beam. In this work by the use of the Timoshenko beam formulation these effects are included as it the use of a two-parameter hysteretic damped foundation. The investigation includes when the beam experiences a moving load with constant or harmonic amplitudes. Hamilton's principle is used to formulate the two governing equations when are then transformed into two algebraic equations by uncoupling and the use of a double Fourier transform w.r.t. moving space and time. The beam deflection is obtained using inverse fast Fourier transform and the solutions obtained are compared with bot Winkler and Pasternak foundation results found in the literature. Calculations are made which investigate the effect of the spatial distribution of the loading and the effects of the beam and foundation properties on the deflected shape, the maximum displacement, critical frequency and the velocity. The results can be used to assess performance and safety aspects of railway and highway structures.
\end{abstract}

Keywords: Timoshenko beam, moving load, hysteretic damping, vibrations.

\section{Introduction}

The topic of moving loads on beams resting on foundations has been studied at length but is still attracting a lot of attention because of the development of very-high speed and capacity vehicles in the mass transportation sector. Also most of the studies found in the literature consider the damping of the foundation to be viscous as opposed to hysteretic, even though hysteretic damping is generally accepted as a major energy dissipation mechanism in soils [1], hence making this type of damping more suitable for modelling foundations. It is also noticeable that studies tend to prefer the simpler EulerBernoulli beam one-equation formulation [2-4] so ignoring shear deformation and rotational inertia.

In addition to studies involving the Euler-Bernoulli formulation and moving loads, others have been carried out using Rayleigh [5], shear [1] or Timoshenko [6-7] formulations, while the foundation has been idealized as Winkler [3], Kelvin [8] or Pasternak [9]. It is important to recognise that the moving load should have a variable amplitude in that the moving loads created by vehicles will have variations in amplitude with time due to many factors such as the roughness of the rail or pavement surface and the mechanical systems of the vehicles.

The objective of this paper is to discuss the dynamic response of a Timoshenko beam on an elastic foundation with hysteretic damping subjected to a moving constant loading and a harmonic moving loading. The two governing equations for the beam/foundation system are formulated using the double Fourier transform with respect to moving time and space. Analyses are then perform to investigate the effect of load distribution, radius of gyration and shear rigidity on the deflected shape, maximum deflection, and critical frequency and velocity.

\section{Formulation and Transformation}

A Timoshenko beam of infinite length resting on a two-parameter foundation with hysteretic damping and subjected to an arbitrary moving load moving in the positive $x$ direction with a velocity $v$ is shown on Fig. 1 


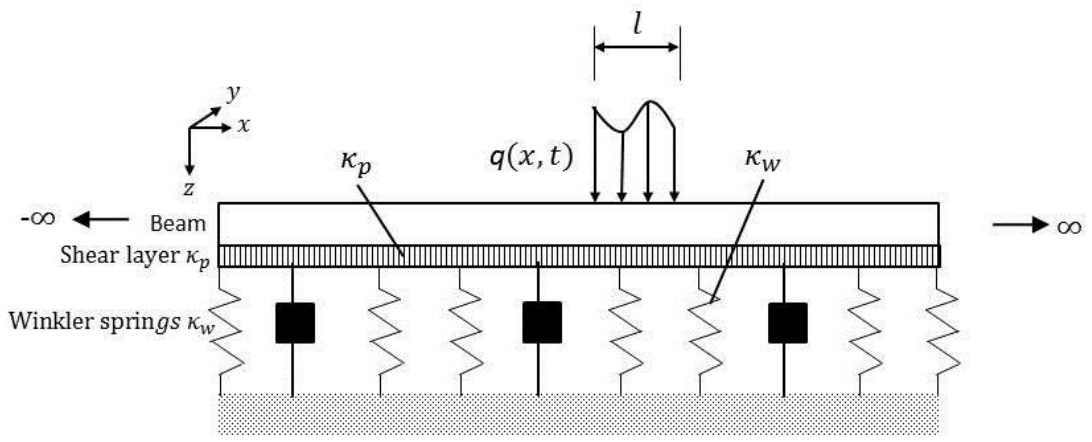

Fig. 1: Infinite Timoshenko beam resting on a two-parameter foundation with hysteretic damping and subjected to an arbitrary moving load.

The vertical displacement and bending rotation of the beam are $w(x, t)$ and $\vartheta(x, t)$ respectively and according to the Hamiltonian principle and Timoshenko beam theory the governing equation of the system can be derived as [7]

$$
\begin{gathered}
\rho A \frac{\partial^{2} w(x, t)}{\partial t^{2}}+S\left(\frac{\partial \vartheta(x, t)}{\partial x}-\frac{\partial^{2} w(x, t)}{\partial x^{2}}\right)+k_{w}(x, t)-k_{p} \frac{\partial^{2} w(x, t)}{\partial x^{2}}+\frac{2 D k_{w}}{\omega} \frac{\partial w(x, t)}{\partial t}=q(x, t) \\
E I \frac{\partial^{2} \vartheta(x, t)}{\partial x^{2}}+S\left(\frac{\partial w(x, t)}{\partial x}-\vartheta(x, t)\right)=\rho A r^{2} \frac{\partial^{2} \vartheta(x, t)}{\partial t^{2}}
\end{gathered}
$$

where $E$ is Young's modulus, $I$ is the cross-sectional moment of inertia, $\rho$ is density, $A$ is cross-sectional area, $S\left(\equiv G k^{*} A\right)$ is the shear rigidity of the beam, $G$ is the shear modulus of the beam, $k^{*} A$ is the effective shear area, $r(\equiv \sqrt{1 / A})$ is the radius of gyration of the beam, $k_{w}$ is the stiffness of the foundation per unit length, $k_{p}$ is the foundation shear coefficient per unit length, $D$ is the damping ratio and $q(x, t)$ is the external load per unit length. The arbitrary moving loading can be defined as

$$
q(x, t)=\phi(x-v t) q_{\phi}(t)
$$

where $\phi(x-v t)$ is the spatial distribution of the load and $q_{\phi}(t)$ is the time history of the load. Also it should be noted that the shear force $Q(x, t)$ can be defined as

$$
Q(x, t)=S\left(\frac{\partial w(x, t)}{\partial x}-\vartheta(x, t)\right)
$$

A moving coordinate system is now introduced as $\eta=x-v t, \widetilde{w}(\eta, t)=w(x, t), \tilde{\vartheta}(\eta, t)=\vartheta(x, t)$, where $\widetilde{w}$ and $\tilde{\vartheta}$ represent the displacement and the rotation of the beam in the moving coordinate system, respectively.

The governing equations can now be written as

$$
\begin{aligned}
\rho A\left(\frac{\partial^{2} \widetilde{w}(\eta, t)}{\partial t^{2}}-2 v \frac{\partial^{2} \widetilde{w}(\eta, t)}{\partial t \partial \eta}\right. & \left.+v^{2} \frac{\partial^{2} \widetilde{w}(\eta, t)}{\partial \eta^{2}}\right)+S\left(\frac{\partial \widetilde{\vartheta}(\eta, t)}{\partial \eta}-\frac{\partial^{2} \widetilde{w}(\eta, t)}{\partial \eta^{2}}\right) \\
& +k_{w} \widetilde{w}(\eta, t)-k_{p} \frac{\partial^{2} \widetilde{w}(\eta, t)}{\partial \eta^{2}}+\frac{2 D k_{w}}{\omega}\left(\frac{\partial \widetilde{w}(\eta, t)}{\partial t}-v \frac{\partial \widetilde{w}(\eta, t)}{\partial \eta}\right)=\phi(\eta) q_{\phi}(t)
\end{aligned}
$$




$$
E I \frac{\partial^{2} \tilde{\vartheta}(\eta, t)}{\partial \eta^{2}}+S\left(\frac{\partial \widetilde{w}(\eta, t)}{\partial \eta}-\tilde{\vartheta}(\eta, t)\right)=\rho A r^{2}\left(\frac{\partial^{2} \tilde{\vartheta}(\eta, t)}{\partial t^{2}}-2 v \frac{\partial^{2} \tilde{\vartheta}(\eta, t)}{\partial t \partial \eta}+v^{2} \frac{\partial^{2} \tilde{\vartheta}(\eta, t)}{\partial \eta^{2}}\right)
$$

The double Fourier transform pair $f(\eta, t)$ and $F(k, \omega)$ are defined as

$$
\begin{array}{r}
F(k, \omega)=\int_{-\infty}^{\infty} \int_{-\infty}^{\infty} f(\eta, t) e^{-i(k \eta+\omega t)} \mathrm{d} \eta \mathrm{d} t \\
f(\eta, t)=\frac{1}{4 \pi^{2}} \int_{-\infty}^{\infty} \int_{-\infty}^{\infty} F(k, \omega) e^{i(k \eta++\omega t)} \mathrm{d} \omega \mathrm{d} k
\end{array}
$$

where $k$ is the transformed domain of moving space and $\omega$ is the transformed domain of time.

On applying the double Fourier transform (Eq. 7) to Eqs. (5) and (6) gives the algebraic equations

$$
\begin{aligned}
&-\rho A\left(\omega^{2}-2 v k \omega+v^{2} k^{2}\right) \widetilde{W}(k, \omega)+ S\left(i k \widetilde{\Theta}(k, \omega)+k^{2} \widetilde{W}(k, \omega)\right) \\
&+\left(k_{w}+k_{p} k^{2}\right) \widetilde{W}(k, \omega)+\frac{2 i D k_{w}}{\omega}(\omega-v k) \widetilde{W}(k, \omega)=\Phi(k) Q(\omega) \\
&-E I k^{2} \widetilde{\Theta}(k, \omega)+S(i k \widetilde{W}(k, \omega)-\widetilde{\Theta}(k, \omega))=-\rho A r^{2}\left(\omega^{2}-2 v k \omega+v^{2} k^{2}\right) \widetilde{\Theta}(k, \omega)
\end{aligned}
$$

where $\Phi(k)$ is the spatial distribution of the load in the transformed wavenumber domain and $Q(\omega)$ is the time history of the loading in the transformed frequency domain.

Using the software package Mathematica $\widetilde{\Theta}(k, \omega)$ can be eliminated from Eqs. (9) and (10) and an expression for $\widetilde{W}(k, \omega)$ can be found as

where the coefficients are

$$
\widetilde{W}(k, \omega)=\frac{\left(c_{1}+c_{2} k+c_{3} k^{2}\right) \Phi(k) Q(\omega)}{c_{4}+c_{5} k+c_{6} k^{2}+c_{7} k^{3}+c_{8} k^{4}}
$$

$$
\begin{gathered}
c_{1}=S-\rho A r^{2} \omega^{2} ; \quad c_{2}=2 \rho A r^{2} v \omega ; \quad c_{3}=E I-\rho A r^{2} v^{2} \\
c_{4}=S k_{w}+2 S i D k_{w}-S \rho A \omega^{2}-k_{w} \rho A r^{2} \omega^{2}-2 k_{w} \rho A i D r^{2} \omega^{2}+\rho^{2} A^{2} r^{2} \omega^{4} \\
c_{5}=2 k_{w} \rho A r^{2} v+6 i D k_{w} \omega r^{2} v-4 \rho^{2} A^{2} \omega^{3} r^{2} v-\left(\frac{2 i D k_{w}}{\omega}\right) S v+2 \rho A \omega^{2} S v \\
c_{6}=E I k_{w}+2 E I i D k_{w}-E I \rho A \omega^{2}-k_{p} \rho A \omega^{2} r^{2}+k_{p} S-\rho A \omega^{2} r^{2} S-k_{w} \rho A r^{2} v^{2}-6 i D k_{w} \rho A r^{2} v^{2} \\
c_{7}=-E I\left(\frac{2 i D k_{w}}{\omega}\right) v+2 E I \rho A \omega v+2 k_{p} \rho A \omega r^{2} v+2 \rho A \omega r^{2} S v+\left(\frac{2 i D k_{w}}{\omega}\right) \rho A r^{2} v^{3}-4 \rho^{2} A^{2} \omega r^{2} v^{3} \\
c_{8}=E I k_{p}+E I S-E I \rho A v^{2}-k_{p} \rho A r^{2} v^{2}-\rho A r^{2} S v^{2}+\rho^{2} A^{2} r^{2} v^{4}
\end{gathered}
$$


Applying the double inverse Fourier transform Eq. (8) to Eq. (11) gives the following expression for beam deflection

$$
W(\eta, t)=\frac{1}{4 \pi^{2}} \int_{-\infty}^{\infty} \int_{-\infty}^{\infty} \frac{\left(c_{1}+c_{2} k+c_{3} k^{2}\right) \Phi(k) Q(\omega)}{c_{4}+c_{5} k+c_{6} k^{2}+c_{7} k^{3}+c_{8} k^{4}} e^{i(k \eta+\omega t)} \mathrm{d} \omega \mathrm{d} k
$$

As this study includes steady-state vibration and the loading is in harmonic form of frequency $\Omega$ with say an amplitude of $\Lambda$ the beam deflection can be expressed as

$$
W(\eta, \Omega)=\frac{\Lambda e^{\Omega t}}{2 \pi} \int_{-\infty}^{\infty} \frac{\left(c_{1}+c_{2} k+c_{3} k^{2}\right) \Phi(k)}{c_{4}+c_{5} k+c_{6} k^{2}+c_{7} k^{3}+c_{8} k^{4}} e^{i(k \eta)} \mathrm{d} k
$$

where the integral is a frequency response function of the system that depends on the loading frequency and properties of the beam and foundation. It should be noted that the closed form of this integral is not always available as the characteristic equation roots cannot sometimes be given in analytical form. Usually the integral is found using IFFT. Also it should be noted that when $r=0, D=0$, and $S \rightarrow \infty$ then the problem reduces to an Euler-Bernoulli beam formulation on a Winkler foundation.

\section{Numerical Results}

In this section validation of the calculation method is first investigated following by solutions involving the effect of load distribution, radius of gyration and shear rigidity on the deflected shape, maximum deflection, and critical frequency and velocity.

\subsection{Validation}

Validation of the current method of solution is first made by comparing with a simplified case [2] where the system is reduced to an Euler-Bernoulli beam formulation resting on a Winkler foundation under a moving point load. The point load is obtained by reducing the width of the loading to infinitely small. As given by Ref. [1] the properties of the beam are $E I=363.35 \mathrm{kN} \mathrm{m}^{2}$ and $m=297.5 \mathrm{~kg} / \mathrm{m}$, and for the foundation the stiffness was $k_{w}=77.17 \mathrm{MPa}$.

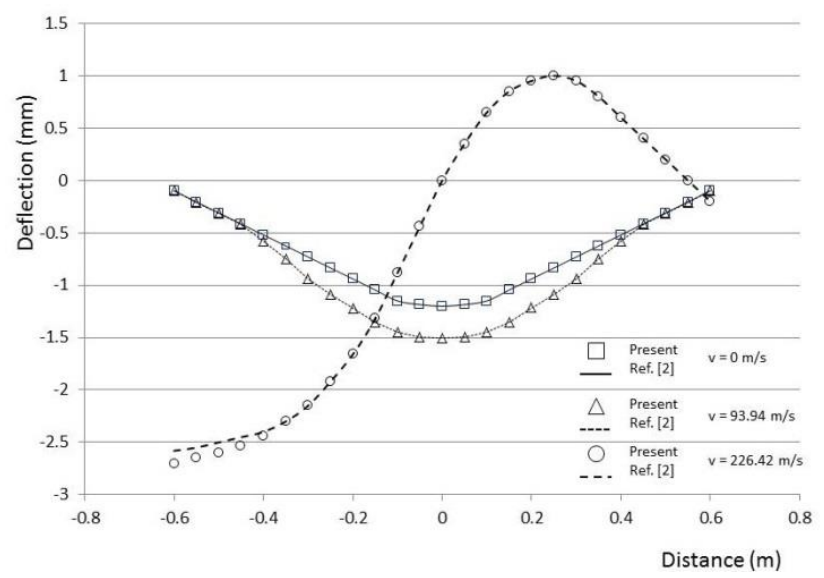

Fig. 2: Comparisons with the results of Ref. [2] for the deflection of an Euler-Bernoulli beam formulation resting on a Winkler foundation and under a moving point load. 
The critical velocity of the reduced system [10]

$$
V_{c r 0}=\sqrt{\frac{2}{\rho A}} \sqrt[4]{E I k_{w}}
$$

is $188.68 \mathrm{~m} / \mathrm{s}$. For comparison three velocities, $0,94.34$ and $226.42 \mathrm{~m} / \mathrm{s}$ are used corresponding to $\alpha=0,0.5$ and 1.2 as described in Ref. [2]. As can be seen from Fig. 2, the current solutions correspond well with those found previously.

\subsection{Effect of Load Distribution}

Two load distributions were investigated both idealised, with one rectangular [1] and the other a triangular [11]. The transformed wavenumber domain of the two distributions are

$$
\Phi(k)= \begin{cases}\frac{2 \Lambda_{m} \sin (k l / 2)}{k}, & \text { rectangle } \\ \frac{4 \Lambda_{m}(1-\cos (k l / 2))}{k^{2} d}, & \text { triangle }\end{cases}
$$

where $\Lambda_{m}$ and $l$ are the maximum value of the load and the width of the load respectively. Taking the same case as in Sect. 3.1 with the exception of assigning 0.1 to $r, 100 \mathrm{MN}$ to $S$. Fig. 3 shows the deflection of the beam subjected to the two types of moving load for a load frequency of $100 \mathrm{~Hz}$, a velocity of $50 \mathrm{~m} / \mathrm{s}$ and hysteretic damping set at $5 \%$. It is clear from the resulting plot that the differing load distributions have little effect on the deflection of the beam.

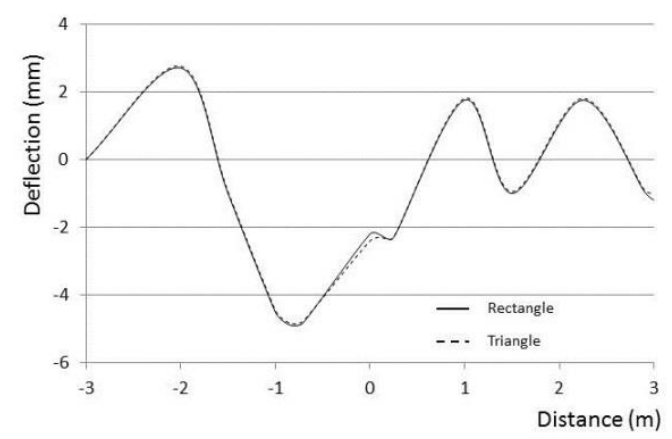

Fig. 3: Deflection of the beam for different load distributions for load frequency of $100 \mathrm{~Hz}, D=5 \%$ and $v=50 \mathrm{~m} / \mathrm{s}$.

\subsection{Loading with Constant Amplitude}

Again following Ref. [1] the properties of the load used here are $l=0.1524 \mathrm{~m}$ and $\Lambda_{m}=262.5 \mathrm{kN} / \mathrm{m}$. Eq. (14) is solved with $\Omega=0$ and the deflected shapes for different properties of the beam are presented followed by the effects of the radius of gyration and shear rigidity on the critical velocity and maximum deflection.

As the beam is considered infinite, the deflection is identical at any time along the moving axis. Fig. 4 shows the beam deflection for a velocity of $50 \mathrm{~m} / \mathrm{s}$ which is lower than the critical velocity of the system. It can be seen that a symmetrical distribution is calculated if no damping is present which is consistent with the findings of Refs. [1] and [5]. Also the maximum deflection increases with decreased shear rigidity and increased radius of deflection, whereas the rest of the beam deflection is almost not affected. 


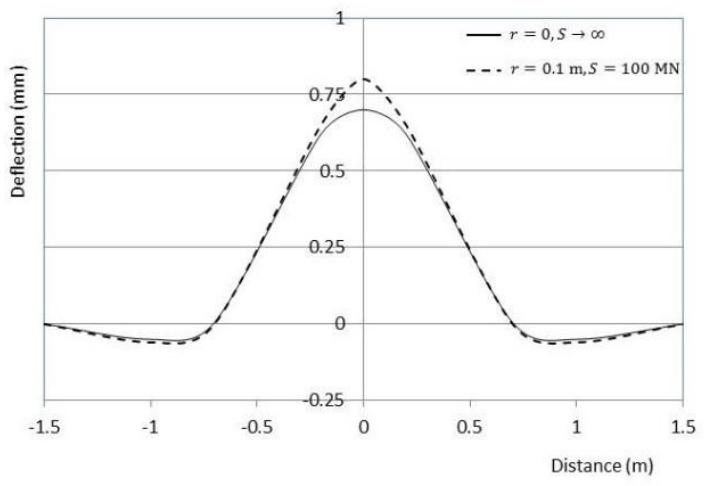

Fig 4: Deflected shape of the beam under a constant loading and with $v=50 \mathrm{~m} / \mathrm{s}$ and $D=0 \%$.

The effects of the radius of gyration and shear rigidity on the critical velocity coefficient are shown on Fig. 5 where the critical velocity coefficient is defined as the critical velocity of the Timoshenko formulation divided by the critical velocity of the Euler-Bernoulli formulation with the same foundation. It can be seen that the critical velocity coefficient decreases with increased radius of gyration and with decreased shear rigidity and the critical velocity ration is much more sensitive to the radius of gyration. The second figure on Fig. 5 shows the variation of the maximum deflection coefficient with the radius of gyration and the shear rigidity. The maximum deflection coefficient is that of a Timoshenko beam divided by that of an Euler-Bernoulli beam with the same foundation.
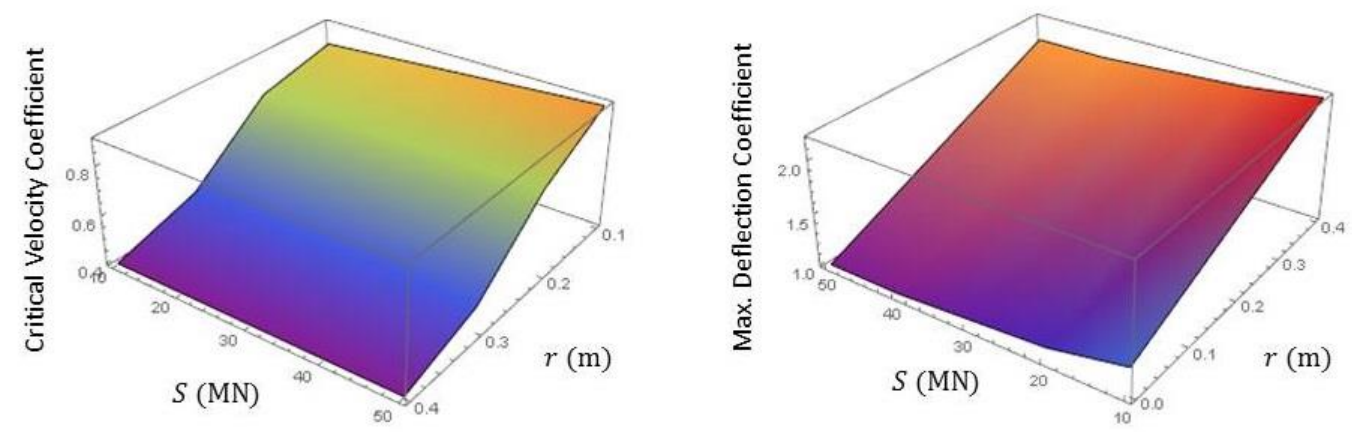

Fig 5: Effects of the shear rigidity and the radius of gyration on the critical velocity coefficient and maximum deflection coefficient when the load is constant and moving.

It can be seen from Fig. 5 that the maximum deflection coefficient increases as the radius of gyration increases and the shear rigidity decreases.

\subsection{Harmonic Moving Load}

Moving with a speed $v$ the maximum deflection due to the harmonic loading vs. the load velocity is shown on Fig. 6 and Fig. 7 for the two frequencies $30 \mathrm{~Hz}$ and $100 \mathrm{~Hz}$ which straddle the critical frequency of the reduced system which is $81 \mathrm{~Hz}$. When the frequency is $30 \mathrm{~Hz}$ two critical velocities occur. Both of the critical velocities are lower that found for the Euler-Bernoulli formulation (the solid line on the Figs.) but however it is clear that the corresponding maximum deflections are higher. When the frequency is increased to $100 \mathrm{~Hz}$ the first critical velocity is difficult to detect whereas the second critical velocity location remains clear. 


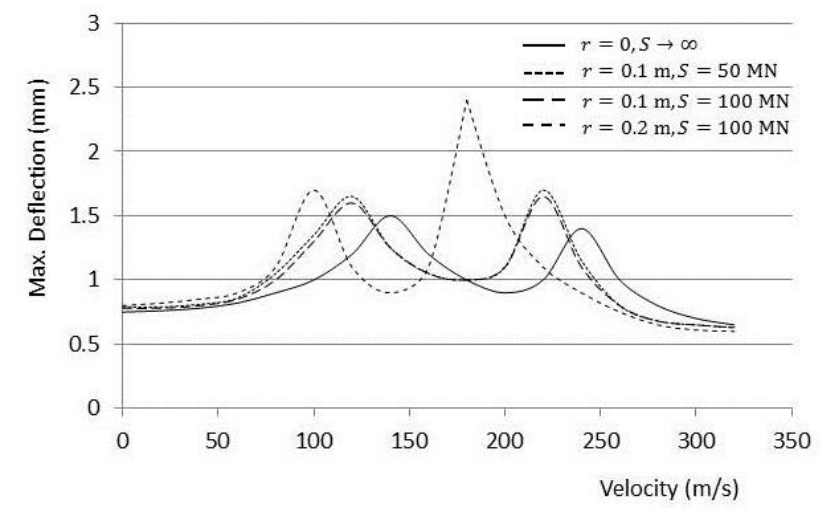

Fig 6: Maximum deflection vs. load velocity for $f=30 \mathrm{~Hz}$ and $D=10 \%$.

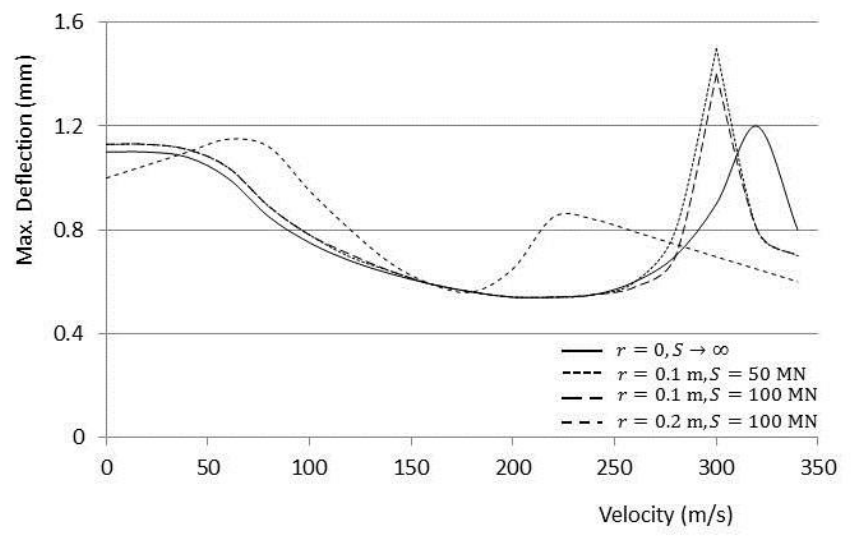

Fig 7: Maximum deflection vs. load velocity for $f=100 \mathrm{~Hz}$ and $D=10 \%$.

Fig. 8 shows the effects of the radius of gyration and shear rigidity on the critical velocity coefficient and the maximum deflection coefficient.
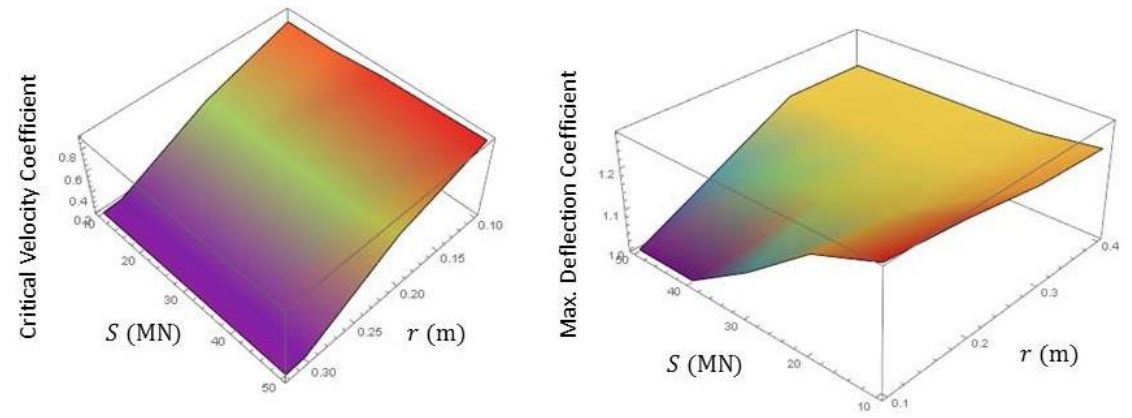

Fig 8: Effects of shear rigidity and the radius of gyration on the first critical velocity coefficient and the maximum deflection coefficient with regard to the first critical velocity with $f=30 \mathrm{~Hz}$ and $D=10 \%$.

For this case the critical velocity coefficient is less than one and the critical velocity coefficient increases as both the radius of gyration and shear rigidity increase. However change in the radius of gyration affects the critical velocity 
coefficient much more than changes in the shear rigidity. Also shown on Fig. 8 is the maximum deflection coefficient with regard to the first critical velocity and its value is higher than one. The variation of the maximum deflection coefficient is not monotonic w.r.t. radius of gyration and shear rigidity. The maximum deflection coefficient strengthens when the shear rigidity is large and the radius of gyration increases but weakens when the shear rigidity is small and the radius of gyration increases.

\section{Conclusion}

The vibration of an infinitely long Timoshenko resting on a hysteretic damped elastic foundation was investigated under moving loads with either constant or harmonic amplitudes. The governing equations were transformed using a doubled Fourier transform w.r.t. moving space and time to the wavenumber and frequency domains. The solution was obtained by using IFFT. It was found that the effect of the spatial distribution of the load on the beam, for those tested, was negligible. Also when the beam is subjected to a moving load with constant amplitude and the velocity is lower than the critical velocity then the beam deflection is symmetric. When the beam is subjected to a harmonic moving load two critical velocity coefficients are generated which significantly increase with the radius of gyration and to a much less extent with increase in the shear rigidity.

\section{Acknowledgements}

This investigation is supported by the Nazarbayev University Small Competitive Grant No. 090118FD5317. The authors declare that there is no conflict of interest and funding associated with this paper.

\section{References}

[1] S. M. Kim and Y. H. Cho, "Vibration and dynamic buckling of shear-columns on elastic foundation under moving harmonic loads." Int. J. Solid Struct., vol. 43, pp. 393-412, 2006.

[2] L. Frýba, Vibration of Solids and Structures Under Moving Loads. (3 ${ }^{\text {rd }}$ ed.) London: Thomas Telford, 1999.

[3] L. Sun, "An explicit representation of steady state response of a beam on an elastic foundation to moving harmonic line loads." Int. J. Numer. Anal. Meth. Geomecha., vol. 27, pp. 69-84, 2003.

[4] H. Yu and Y. Yuan, "Analytical solution for an infinite Euler-Bernoulli beam on a viscoelastic foundation subjected to arbitrary dynamic loads.” J. Eng. Mech., vol. 140, pp. 542-551, 2013.

[5] S. M. Kim, "Stability and dynamic response of Rayleigh beam-columns on an elastic foundation under moving loads of constant amplitude and harmonic variation." Eng. Struct., vol. 27, pp. 869-880, 2005.

[6] S. Verichev and A. Metrikine, "Instability of a bogie moving on a flexibly supported Timoshenko beam." J. Sound Vib., vol. 253, pp. 653-668, 2002.

[7] Y. H. Chen, Y. H. Huang and C. T. Shih, "Response of an infinite Timoshenko beam on a viscoelastic foundation to a harmonic moving load." J. Sound Vib., vol. 241, pp. 809-824, 2001.

[8] A. Wolfert, H. Dieterman and A. Metrikine, "Stability of vibrations of two oscillators moving uniformly along a beam on a viscoelastic foundation." J. Sound Vib., vol. 211, pp. 829-842, 1998.

[9] M. Ahmadian, R. Jafari-Talookolaei and E. Esmailzadeh, "Dynamics of a laminated composite beam on Pasternakviscoelastic foundation subjected to a moving oscillator." J. Vib. Cont., vol. 14, pp.807-830, 2008.

[10] S.-M. Kim and J. M. Roesset, "Dynamic response of a beam on a frequency-independent damped elastic foundation to moving load." Can. J. Civil Eng., vol. 30, pp. 460-467, 2011.

[11] F. Wang, "Mechanistic-empirical study of effects of truck tire pressure on asphalt pavement performance." Ph.D. dissertation, The University of Texas at Austin, Texas, 2005. 